Original Research Paper

\title{
Adsorption and Distribution of Selected High Molecular Weight Polycyclic Aromatic Hydrocarbons in a Typical Coastal Sand Deposit
}

\author{
Uzoije Atulegwu Patrick and Ezenobi Chukwunoye Christain \\ Department of Environmental Technology, School of Environmental Science, \\ Federal University of Technology, Owerri, Imo State, Nigeria
}

\author{
Article history \\ Received: 24-02-2018 \\ Revised: $12-03-2018$ \\ Accepted: 27-03-2018 \\ Corresponding Author: \\ Uzoije Atulegwu Patrick \\ Department of Environmental \\ Technology, School of \\ Environmental Science, \\ Federal University of \\ Technology, Owerri, Imo State, \\ Nigeria \\ Email: atulegwuuzoije@yahoo.com
}

Abstract: Contaminant migration study through soil medium is a veritable tool for a match towards sustainable groundwater protection. It is also essential to predict the fate and Transport of pollutants and to evaluate pollution risks. In this study, migration and distribution of High Molecular Weight (HMW) Polycyclic Aromatic Hydrocarbons (PAHs) through a coastal sand deposit of a wetland was investigated. Flow simulation of the PAHs to determine the migration of the selected HMW was carried out by soil column method. With the help of gravity, the PAHs polluted wastewater collected from the study site was made to pass through a soil column. The analysis of the pore water collected as exit solution showed apparent concentration differences of various selected (HMW) PAHs from the original concentrations. The relative concentrations with respect to pore water assumed dome curves in which various selected (HMW) PAH concentrations rose gradually to the peak then decreased and tailed down with increasing pore volume effluents. The observed concentration differences was attributed to adsorption. Concentration values of the PAHs adsorbed onto the soil is of the following order; $\operatorname{Benzo}(b)$ flouranthene $>\operatorname{Benzo}(\mathrm{K})$ flourathene $>1,2,3-\mathrm{cd}$ pyrene $>$ Dibenzo(a,h)anthrene $>$ Benzo(a)pyrene $>$ Chrysene $>$ Benzo(a)anthrene. Distribution of the selected (HMW) PAHs within the soil column was also investigated. There was significant percentage asymmetric fixation of Benzo(b)flouranthene, Benzo(a)pyrene, Chrysene, 1,2,3-cd pyreneand Benzo(K)flourathene at the upper segment (0-4 and 4$9 \mathrm{~cm})$ of the soil column. On the other hand, retentions of Dibenzo(a,h)anthrene and Benzo(a)anthrene were equally significant at the lower segment $(9-15 \mathrm{~cm})$. Despite the asymmetric fixation of Benzo(a)pyrene and Chrysene at the upper segment of the column, their concentrations and that of Benzo(a)anthrene) compound were significant in the exit solution. However, Migration and distributions of the HMW PAHs on the soil under investigation were essentially the functions of their chemical structures, solubility and to an extent the molecular weights. Given the distribution and migration pattern of the selected (HMW), Dibenzo(a,h)anthrene, Benzo(a)pyrene, Chrysene and Benzo(a)anthrene have great potentials to migrate further down the subsurface and to the aquifer to compromise the integrity of the groundwater in the wetland.

Keywords: PAHs, Contaminant Migration, Soil, Adsorption, Distribution, HMW, Groundwater, Porous Medium 


\section{Introduction}

Contaminant migration through porous media is largely determined by two major factors; characteristics of the contaminants and that of the media (Haws et al., 2004). Molecular mass, chemical structure, volatility, density, solubility etc of the contaminants essentially decide the extent to which the contaminants move and distribute through the media pores and on the media respectively. Composition, nature and water hydrological process of the media also control migration of the contaminants. Polycyclic Aromatic Hydrocarbons (PAHs) have a wide range of sources which include; oil, coal and tar deposits, byproducts of fossil fuel/biomass combustion etc. Given its availability via natural and anthropogenic sources, PAHs are ubiquitous in the environment, particularly in soil environment. This informed the United State Environmental Protection Agency (USEPA, 1990) compilation and categorization of $16 \mathrm{PAHs}$ as being priority pollutants in wastewater while 24 others are in soil and sediment (Christensen and Arora, 2006). Each $\mathrm{PAH}$ compound exhibits various properties with respect to transformation, biodegradation, transportation, lipophilicity/hydrophobicity etc. As a result, the PAHs go through different levels of migration mechanism outlined above. In soil environment, transformation of solute substances to other forms is likely. In most cases, substances form complexes with the organic matter and the chemical contents of the soil (van Genuchten and Wagenet, 1989). The resultant compound(s) persist in the environment depending on their stability. The extents to which various PAH compounds react and reduced by the activities of micro-organism vary. The rate of biodegradation and transport of multi-cyclic compounds such as PAHs in the soil medium increases with decrease in number of rings and molecular masses. Processes like sorption, distribution, retardation affect the transport, bioavailability and biodegradability of PAH compounds (Ortega-Calvo et al., 1997; Piatt et al., 1996). These processes are essentially dependent on the hydrophobicity of various PAHs and the measure of organic substance and pore sizes of the soil medium (Chiou et al., 1998). In most cases, organic partition coefficient in water $\left(K_{O W}\right)$ measures the hydrophobicity/lipophilicity of hydrophobic sorption (Lohmann, 2003). Also, the distribution coefficient (KD) of a solute on a solid phase increases with increasing hydrophobicity of the solute and the organic content of the soil (Yaron et al., 1996). Therefore, the interaction of the PAHs with soil and segments is to a large extent predicated on the organic carbon. This corroborates the established fact of organic carbon being the most sorbent of hydrophobic organic pollutants in soil or segment if $f_{o c}>0.1 \%$ (Nguyen and Ball, 2006). However, at low organic partition coefficient $\left(f_{o c}\right)$ i.e., $f_{o c}<0.1 \%$ which is peculiar to most aquifer sands, sorption of PAHs can still be realized due to partitioning onto the organic matter and the minerals (Joo et al., 2008). Most PAH compounds, especially the high molecular ones persist on the soil surface and subsequently bio-accumulate in the organisms and plant roots (He et al., 2006). The level of retardation during migration, persistence and distribution on the soil are indeed functions of the properties of soil and PAH compounds. However, cases of groundwater contamination of lighter PAH compounds through lateritic and clayey formations have been reported, (O'Reilly et al., 2001; Lee et al., 1992) but no much research work has been reported on distributions of high weight molecular PAH on soil and possible transport to aquifer.

Previous studies have considered the sorption and distributions of naphthalene, anthracene and fluoranthene on soils and sediments of alluvial composition (Toul et al., 2003; Park and Juan, 2000). Percentage of sorption of PAHs was more in sediment than in the soil and the magnitude of sorption was in this order; sediment $<$ soil and naphthalene $<$ phenanthrene $<$ pyrene, respectively (Hwang et al., 2003; Hiller et al., 2008). Effects of temperature on the distribution of naphthalene, phenanthrene and pyrene on the soil and sediment (Hiller et al., 2008) has also been reported. Little has been reported on the distribution of HMW PAHs on the soil and on their tendency to ground water pollution. The study area is a part of coastal region which houses many asphalt processing plants of varying sizes. Each plant has a dump site within and around area where the remnants of the produced asphalts are usually deposited. Through surface runoff and overland flow the asphalt remnants are washed into water bodies and also get into subsurface through infiltration. PAH molecules which are known active ingredients of asphalts, especially the high molecular weight ones migrate down the subsurface to pollute the groundwater (Lee et al., 1992; Olsen and Davis, 1990; Hwang and Cutright, 2002; Weber et al., 1992; Edwards, 1983). Previous studies have shown that a good amount of Low Molecular Weight (LMW) components of PAH on the ground surface evaporate into the air at high temperature leaving behind the high molecular ones (Hiller et al., 2008). Given the high vulnerability of groundwater pollution by the high molecular weight PAH compounds, possible health issues associated with the compounds and the peculiarities of the coastal terrain, the study of the transport and distributions of the high weight molecular $\mathrm{PAH}$ on the coastal sand deposit to ascertain their affinity levels to the soil as they migrate down subsurface and their potentials to pollute groundwater become very expedient. The aim of this work is therefore 
to study the distribution of the HMW PAHs compounds of the 16 most commonly evaluated unsubstituted PAHs on the typical coastal sand deposit of the wetland land. This study which will bring to fore the distribution pattern of the HMW PAHs on such a coastal deposit, will determine the groundwater pollution potential by each selected HMW PAHs.

\section{Materials and Methods}

\section{Soil Property Characterization}

Soil samples were collected from a location outside the asphalt dump site in the study area (Oyigbo, South-South Nigeria). The samples which were collected at the depth of between 20-60 cm were, air-dried, homogenized, screened to remove debris before they were analyzed for porosity, bulk density, particle density, hydraulic conductivity and organic carbon at the soil laboratory of the Institute for Erosion Studies of Federal University of Technology Owerri. To ascertain the level of natural concentrations of the HMW PAHs of interest in the soil sample, analysis of the hydrocarbons was equally carried out on the soil. The analysis was carried out in three replicates using standard methods.

\section{Column Experiment}

A column of internal diameter of $2.5 \mathrm{~cm}$ and a length of $42 \mathrm{~cm}$ was packed with a sample of undisturbed soil samples collected, pressed down to reflect the field situation. Porous stainless plates of 90 and $1 \mu \mathrm{m}$ were placed on the top and bottom of the column respectively to ensure even distribution of the solution. The entire setup was placed on a balance to monitor the soil water content throughout the experiment. This set-up was made in two sets; one set-up was to determine the pore water velocity of the soil and the other was to determine the flow properties of the PAHs. A known concentration of solution of non-adsorbing substance $(\mathrm{NaCl})$ was introduced into one of the set-ups to determine the porewater velocity of the coastal sand by collecting and analyzing the exit solution at 18-30 days Thereafter, known initial concentrations of the solution of PAH collected from the wetland was introduced into a reservoir positioned above the column of the other setup. The exit solution was also collected with time and analyzed to ascertain the effluent concentrations of the selected PAHs using Gas Chromatography (HP 5890 II, Hewlett-Packard Co., Inc) with a Flame Ionization Detector (GC-FID) and columns. The solution was allowed to flow into the soil column at constant velocity through gravity and with help of the pump. After a while, the solution started dripping from the exit end of the column, the effluent was collected for analysis on daily basis between 18-30 days to determine the concentrations of various $\mathrm{PAH}$ compounds at breakthrough using the aforementioned method. Each analysis took place in three replicates. The amounts of PAHs adsorbed to the sorbent were calculated by the difference between the initial and effluent concentrations of the PAHs. Volume of the effluent which represented the pore volume, was equally monitored for the same number of days. Moreover, weight of the column was read to determine the water content of the column material. Concentrations of the PAH compounds under study were analyzed from each effluents collected. At the end of the column discharge, the outer surface of the soil column was marked in three places at 4,5 and $6 \mathrm{~cm}$ interval to represent top, middle and bottom layer respectively. Each soil column segment was subjected to PAH analysis through solvent extraction and analysis using gas chromatographic method. Finally, the data obtained were evaluated by the sorption models.

\section{Model Description/ Data Analysis}

Sorption models applied to determine the distribution of PAHs on coastal sand are described as follows:

Velocities of the non-adsorbing substance and PAHs were determined by dividing the column length by the mean resident time expressed as:

$\frac{l}{t_{m}}$

where, $l$ and $t_{m}$ represent the length of the column and mean resident time respectively.

$t_{m}$ is in turn expressed as:

$t_{m}=\frac{\int_{t_{\min }}^{t_{\max }} t C d t}{\int_{t_{\min }}^{t_{\max }} C d t}$

Where:

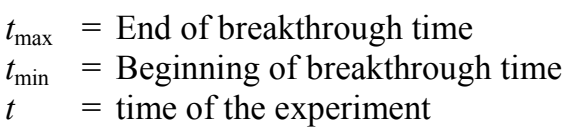

$t_{m}$ is obtained for the nonadsorbing substance and PAHs to calculate pore-water velocity $\left(V_{P}\right)$ and the migration velocity of PAHs through the column $\left(V_{C}\right)$ respectively. With $V_{P}$ and $V_{C}$, retardation factor, $R_{f}$ which measures the reluctance at which PAHs migrate through the soil pores due to small pore size and affinity to the soil matrix, was determined using the following model:

$R_{f}=\frac{V_{P}}{V_{C}}$ 
Then, $K_{d}$ was calculated directly from the retardation factor, $R_{f}$ with values of the soil properties which is expressed as:

$R_{f}=1+\frac{K_{d} \rho_{b}}{n}$

Where:

$\rho_{b}=$ Soil bulk density

$n=$ Soil porosity

Convincing evidences have been reported for the dominant role organic carbon plays in the sorption of PAHs onto soil. It implies that the organic carbon content of the soil essentially determine the sorption rate of $\mathrm{PAH}$ compounds on the soil. Therefore, organic carbon partition coefficient $\left(K_{O C}\right)$ was calculated with this model:

$K_{d}=f_{o c} K_{O C}$

where, $f_{o c}$ represents the fraction of organic carbon in the soil.

\section{Results}

\section{Physical and Chemical Properties of HMW PAHs}

Properties of the selected HMW PAH compounds are shown on Table 1 as presented by Chen et al.
(2006). They are hydrocarbon compounds of high molecular weight with carbon rings of between 4-6. They have varying molecular weights, structures and solubility. They are generally insoluble though some are more soluble than the others. In terms of chemical structure, they are all angularly fused, some are alternate. i.e., planer and symmetric and nonalternate PAH compounds, Benzo(b)flouranthene, Benzo(K)flouranthene and Indeno1,2,3-cd pyrene are nonalternate $\mathrm{PAH}$ compounds while Benzo(a)anthrene, Chrysene, Benzo(a)pyrene and Dibenzo(a,h)anthrene are alternate given their planer and symmetric structural patterns. The alternate PAH compounds seem to require high energy of solubilization hence less soluble than the non-alternate PAH compounds.

Results of The average initial concentrations of the selected HMW PAH compounds analyzed from the water samples of the wetland are presented in Table 2. The result shows different concentration values for various PAH compounds. From the result, Indeno(1,2,3-cd)pyrene has the highest value, followed by Benzo(K)flourathene with Benzo(a)anthrene having the lowest value. The PAH concentrations in the wetland are well above the recommend value meant for the environment.

Results of the soil analysis representing the soil characteristics of the study area are shown on Table 3. The average values of the soil parameter analyzed reflect that of a typical coastal area (Chiou et al., 1998).

Table 1: Properties of the selected PAHs used in the experiment

\begin{tabular}{lllll}
\hline PAHs & Ring & Molecular formula & Molecular weight & Solubility \\
\hline Benzo(b)flouranthene & 5 & $\mathrm{C}_{20} \mathrm{H}_{12}$ & 252.32 & 0.0020 \\
Benzo(K)flourathene & 5 & $\mathrm{C}_{20} \mathrm{H}_{1}$ & 252.32 & 0.0008 \\
$1,2,3$-cd pyrene & 6 & $\mathrm{C}_{22} \mathrm{H}_{12}$ & 276.34 & 0.0005 \\
Dibenzo(a,h)anthrene & 5 & $\mathrm{C}_{22} \mathrm{H}_{12}$ & 278.36 & 0.0006 \\
Benzo(a)pyrene & 5 & $\mathrm{C}_{20} \mathrm{H}_{12}$ & 252.32 & 0.0060 \\
Chrysene & 4 & $\mathrm{C}_{18} \mathrm{H}_{12}$ & 228.3 & 0.0020 \\
Benzo(a)anthrene & 4 & $\mathrm{C}_{18} \mathrm{H}_{12}$ & 228.3 & 0.0090 \\
\hline
\end{tabular}

Source; Chen et al. (2006)

Table 2: Average Initial concentrations of the selected HMW PAH compounds

\begin{tabular}{ll}
\hline HMW PAH compounds & Initial concentrations (mg/l) \\
\hline Benzo(b)flouranthene & 0.30824 \\
Benzo(K)flourathene & 0.95644 \\
Indeno(1,2,3-cd)pyrene & 2.14836 \\
Dibenzo(a,h)anthrene & 0.24863 \\
Benzo(a)pyrene & 0.19886 \\
Chrysene & 0.34315 \\
Benzo(a)anthrene & 0.09845 \\
\hline
\end{tabular}

Table 3: Average values of the soil properties

\begin{tabular}{lllll}
\hline Porosity $(\%)$ & Bulk density $\left(\mathrm{gcm}^{-3}\right)$ & Hydraulic conductivity $\left(\mathrm{cms}^{-1}\right)$ & Particle density $\left(\mathrm{gcm}^{-3}\right)$ & Organic carbon $(\%)$ \\
\hline 29.1 & 1.49 & 0.0202 & 2.6 & 1.27 \\
\hline
\end{tabular}




\section{Sorption and Distributions}

Results of HMW PAHs analyzed after the column experiment showed in detail the level of affinity of the selected PAH to the soil under study. This was corroborated by the apparent changes in the concentration of the PAHs of the exit solution from the column. The changes were shown in the apparent reductions in the values of PAHs concentration in the exit solution from the end of the column, although the results are not shown on the table for brevity. However, the reduction in concentrations of Various HMW PAH compounds on the exit solution took place in this order Benzo(a)anthrene $<$ Dibenzo(a,h)anthrene $<\quad$ Benzo(a)pyrene $<\quad$ Chrysene $<$ Indeno(1,2,3-cd)pyrene $<\quad$ Benzo(K)flourathen $<$ Benzo(b)flouranthene. On the sorption effect, PAH compounds exhibited different sorption affinities to the coastal sand deposit and by extension have different exit concentrations and breakthrough periods.

Figure 1 represents various concentration profiles of the $\mathrm{PAH}$ compounds in relation to the pore volumes collected as effluent. The figure shows that the PAHs plume assumed different gradients of dome curves wherein the concentrations rose gradually to the peak, then decreased and tailed down with increasing pore volume effluents. PAH compounds of different concentrations made varying breakthrough with various pore water volumes. Benzo(a)anthrene and Chrysene made significant breakthrough at relative concentration of 1 in low pore water volume of $2,5 \mathrm{~cm} 3$ and the trend of relative concentration decrease with high pore water volume was observed as follows; Benzo(a)anthrene- ChryseneBenzo(a)pyrene - Benzo(K)flourathen- Indeno(1,2,3cd) pyrene $=$ Dibenzo(a,h)anthrene $=$ Benzo(b)flouranthene . The seeming varying PAHs plume concentration distributions resulted to different steepness of the curves. From the figure, benzo(b)flouranthene is the least steep, least skewed from the center and showed the most significant tailing of the breakthrough curves. On the other hand, Benzo(a)anthrene breakthrough curve shows the most steep and short tailing. Basically, steepness and tailing down of the PAH plume curves(breakthriough) decreased as follows; Benzo(a)anthrene $>$ Chrysene> Benzo(a)pyrene $>\quad B e n z o(K)$ flourathen $>\quad$ Indeno(1,2,3cd)pyrene> Benzo(b)flouranthene.

Figure 2 depicts the relative concentrations of Various HMW PAH compounds with breakthrough time. The figure represents the breakthrough times of the compounds wherein the breakthrough period increased with time and remained constant after certain times. From the figure, Benzo(b)flouranthene had most delayed breakthrough while Benzo(a)anthrene experienced the quickest breakthrough period. Breakthrough times remained constant at 15 days and the time increased to 30days for the HMW PAH understudy. Breakthrough-time constant values increased down the HMW PAH compounds as follows; Benzo(a)anthrene Chrysene> Benzo(a)pyrene> Benzo(K)flourathen> Indeno(1,2,3-cd)pyrene> Benzo(b)flouranthene respectively.

Table 4: Values of the sorption model parameters

\begin{tabular}{|c|c|c|c|c|c|c|c|}
\hline & $\begin{array}{l}\text { Benzo(b) fluo } \\
\text { Co }=0.30824\end{array}$ & $\begin{array}{l}\text { Benzo(k)fluo } \\
\text { Co }=0.95644\end{array}$ & $\begin{array}{l}1,2,3 \text {-cd pyrene } \\
\mathrm{Co}=2.14836\end{array}$ & $\begin{array}{l}\text { Dibenzo(a,h) } \\
\text { anthrane } \\
\mathrm{Co}=0.24863\end{array}$ & $\begin{array}{l}\text { Benzo(a)pyrene } \\
\text { Co }=0.19886\end{array}$ & $\begin{array}{l}\text { Chrysene } \\
\text { Co }=0.34315\end{array}$ & $\begin{array}{l}\text { Benzo(a)anthrane } \\
\text { Co }=0.19886\end{array}$ \\
\hline $\begin{array}{l}\text { PAHs Sorption } \\
\text { amounts } \mu g / g \text { soil }\end{array}$ & 0.2697 & 0.9490 & 1.2550 & 0.2299 & 0.1531 & 0.1947 & 0.0212 \\
\hline$K_{d}$ & 7.1800 & 9.1400 & 4.3200 & 6.0600 & 3.4190 & 4.0500 & 0.3130 \\
\hline$\%$ adsorption & 87.5000 & 99.2200 & 58.5500 & 92.4700 & 76.9900 & 56.7400 & 10.6600 \\
\hline$K_{o c}$ & 0.2124 & 0.7472 & 0.2882 & 0.1810 & 0.1206 & 0.1533 & 0.0167 \\
\hline$R_{f}$ & 1.3679 & 1.4679 & 1.1073 & 1.3103 & 1.0790 & 1.2074 & 1.0160 \\
\hline
\end{tabular}

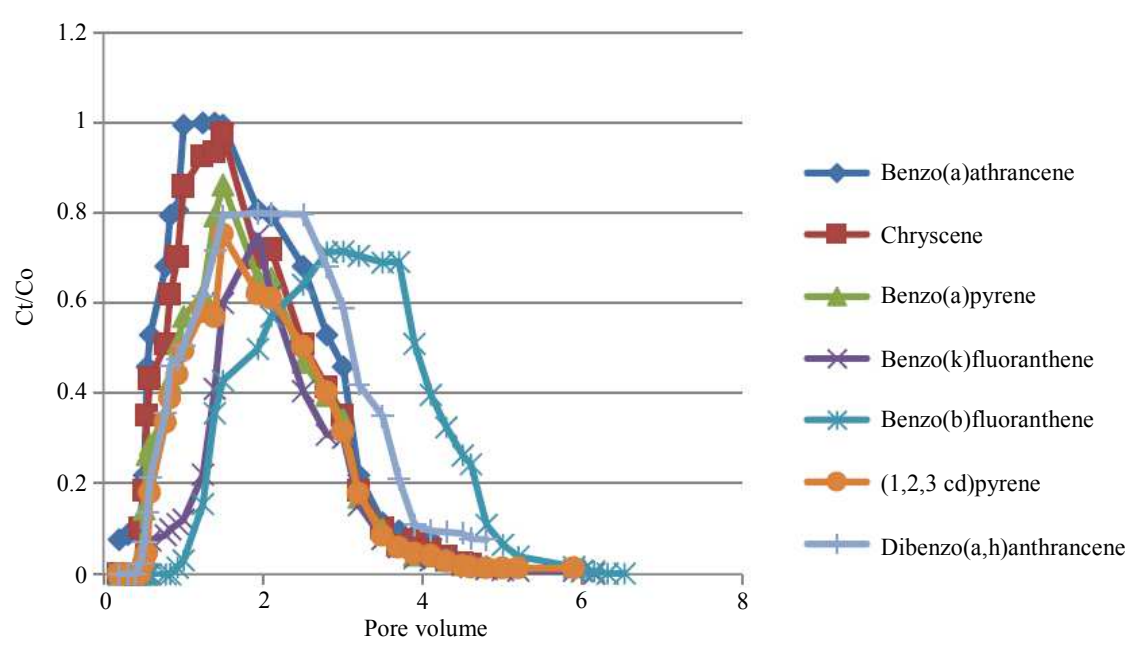

Fig. 1: Breakthrough curves for different PAH compounds 

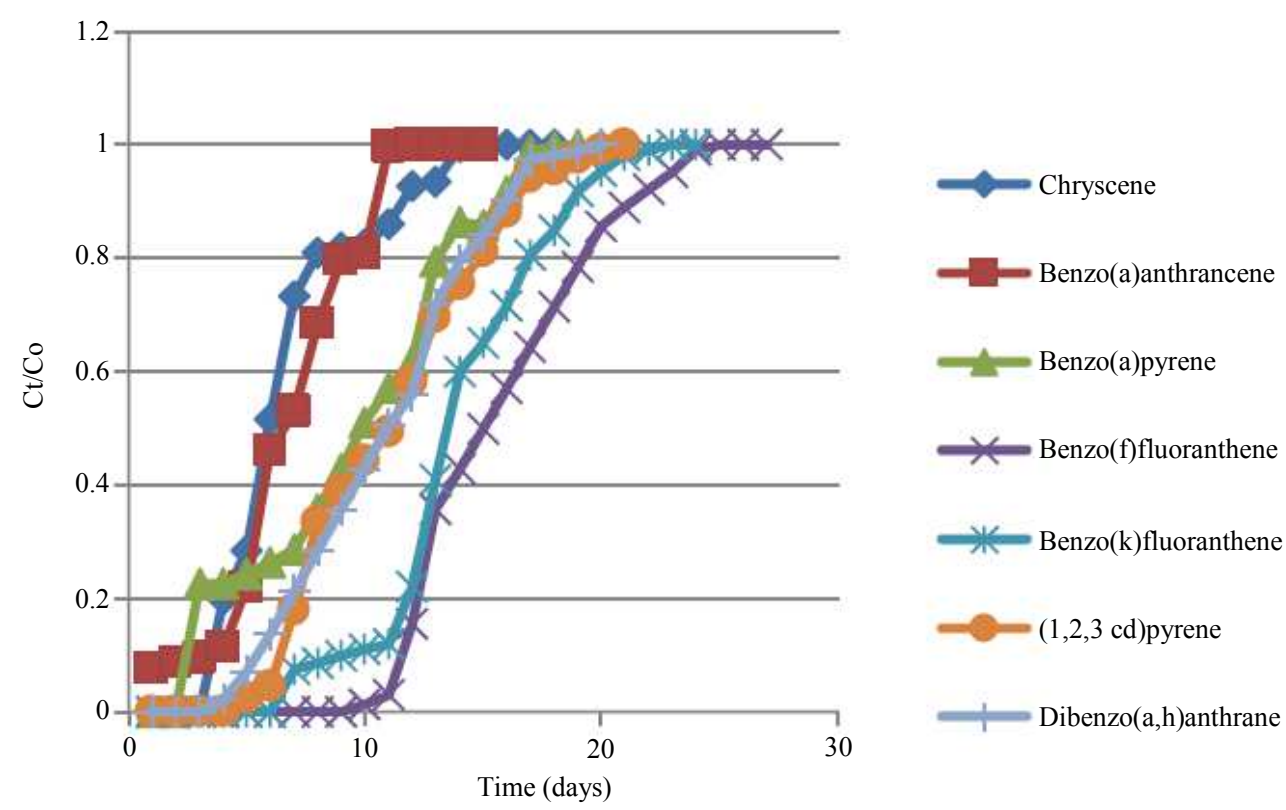

Fig. 2: Breakthrough curves of various $\mathrm{PAH}$

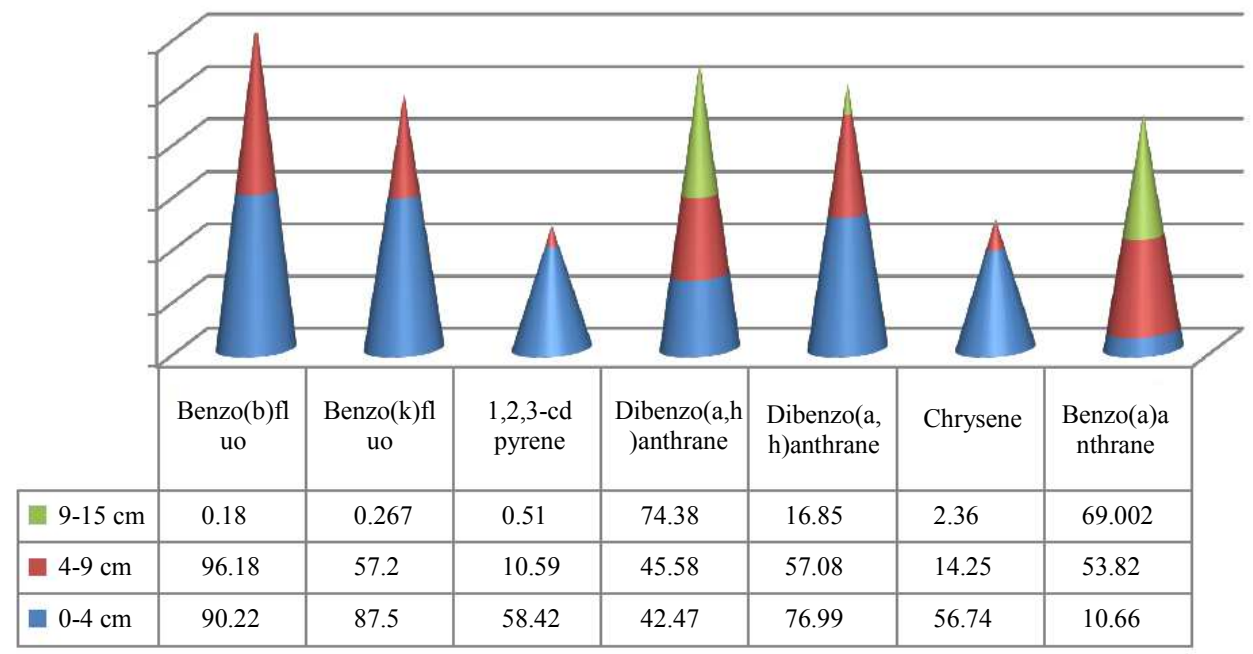

Fig. 3: PAHs'distribution on the soil column

To ascertain the level of PAH distribution of the soil medium across the soil column, the column was divided into top, middle and bottom segments and soil analysis of each segment was carried to determine the concentration PAHs distribution. The percentage distribution of various PAH compounds at each segment are presented on Fig. 3. PAH compounds were retained at different values in each segment of the column. At the last segment (between 9-15 cm) of the column, Benzo(b)flouranthene was least retained whereas benzo(a)anthrene and Dibenzo(a,h)anthrene were most retained. Amounts or percentage of adsorption on the soil increased with the increase in the molecular weights and structure of various PAHs. This is apparent on the sorption values of Dibenzo(a,h)anthrene and benzo(K)flourathene. The sorbed amounts of those compounds increased with their molecular weights. Looking at figure, amount and percentage of adsorption of Dibenzo(a,h)anthrene is less than that of benzo(K)flourathene despite its (Dibenzo(a,h)anthrene) high molecular weight.

Also, Parameters of the sorption model of the study were evaluated and presented on Table 4 . Values of the model parameters appear to be in correlation with the physical and chemical characteristics of the PAHs understudy. The $K_{O C}, K_{d}$ and $R_{f}$ values of the PAHs 
increased with corresponding increase in the values of the molecular weights and structure except 1,2,3-cd pyrene and Dibenzo(a,h) anthrane.

\section{Discussion}

The reduction in the concentration values of $\mathrm{PAH}$ found in the exiting solution(effluent) confirm that sorption process took place. In effect, some amounts of PAHs adsorbed on the soil matrix. The apparent variations in the concentration reduction of the PAH compounds is most likely the effects of different chemical and physical properties exhibited by Various HMW PAH compounds such as the molecular structure, molecular mass, solubility etc (Joo et al., 2008). Consequent upon that, the $\mathrm{PAH}$ compounds made considerable contacts of varying degrees with the soil column, which led to the capricious breakthrough of various compounds (Anderson et al., 2000; Kim and Osako, 2003). The relative concentration profile the PAH compounds measured in relation to the pore water volume showed dome curves of various steepness. The steepness of the curve for the PAHs increased in the following trend; benzo(b)flouranthene $<$ benzo(K)flourathene $<1,2,3$-cd pyrene $<$ Dibenzo(a,h)anthrene $<$ benzo(a)pyrene $<$ Chrysene $<$ benzo(a)anthrene. key factors such as breakthrough amount in relation to the pore water volume, the extent of contact with the medium and sorption amount, determine the shape of the breakthrough curve contaminants (Allen-King et al., 2002; He et al., 2006). The significant breakthrough at low pore water volume was a consequence of insufficient contact time and by extension low sorption. These processes led to high steep curve and short tailed curves. On the contrary, an insignificant breakthrough of the transporting contaminant with high pore water volume implies good contact time and an appreciable amount of sorption of the contaminant on the solid medium (Zhou and Wang, 2013). The structure of such curves is usually low steeped gradient with long tailed (Kim and Osako, 2003). In this study, benzo(b)flouranthene plume curve is most tailed-down and the least steep because it made significant contact time with the coastal sand medium and also adsorbed most on the medium. On the other hand, the short tailing and high steep curve observed on the Benzo(a)anthrene plume could be attributed to less contact with the soil medium and by implication less sorption amount and quick breakthrough. Different breakthrough pattern which describes sorption amounts showed curves of varying skewness and indeed a measure of asymmetry around the mean point of the breakthrough curves (Zhou and Wang, 2013). This is obvious in the breakthrough curves on Fig. 1. The measure of asymmetry varies for different $\mathrm{PAH}$ compounds under study which was occasioned by differences in migration time measured in the pore water volume. Asymmetry tail for Benzo(b)flouranthene, benzo(K)flouratheneand 1,2,3-cd pyrene dragged toward increasing pore volume, but curves for Dibenzo(a,h)anthrene benzo(a)pyrene Chrysene and benzo(a)anthrene skewed slightly. What this means is that the former PAH compounds mentioned experienced delayed breakthrough due to large contact made with the soil matrix. It is also a possibility that the compounds were in Fickain regime having transverse a large distance and considerable long time through the soil column (Park and Juan, 2000). The latter group of PAH compounds have apparently short asymmetric tails occasioned by quick breakthrough, (van Genuchten and Wagenet, 1989; He et al., 2006; Ma et al., 2007).

In relation to the relative concentration with breakthrough time, the time of breakthrough of the PAH compounds became constant at different breakthrough times. HMW PAH compounds which exhibited less affinity to the coastal sand medium, experienced early constant breakthrough time, hence made little contact time with the medium. What this meant was that such compounds had early breakthrough periods with substantial amounts breaking through the coastal sand column. Breakthrough times of Benzo(a)anthrene and Chrysene were constant at earlier periods than the other $\mathrm{PAH}$ compounds because there was apparent low sorption occasioned by insufficient contact time with the coastal sand. Generally, the prevailing breakthrough curve pattern suggests that the breakthrough time for the $\mathrm{PAH}$ components as arranged, decreased accordingly with the corresponding decrease in contact time. With the existing situation under consideration, amount of PAHs adsorbed onto the coastal sand matrix decreased in the same order. The steepness of the breakthrough curves in relation to quick and delayed breakthrough time, the time of contact with the coastal sand matrix with consequent amount adsorbed onto the soil are based on the solubility and molecular weights (Patrick and Cosmas, 2016), (Uzoije et al., 2015) of the substances. A sparingly soluble (weak solvation) substance is readily available in solution, in so doing, likely to adsorb or distribute on the medium as it migrates through a porous medium (Benekos et al., 2006). Also, low solubility of highly hydrophobic PAHs in the solution, constitute long term contamination source to the soil whereas the more soluble ones constitute more danger to groundwater (ThAbdel-Moghny et al., 2012). Moreover contaminants of high molecular weight also have the tendency to adsorb easily onto the medium because the largeness of the molecular structure does not permit free molecular movement through the medium pores (Anderson et al., 2000). Benzo(b)flouranthene,Benzo(K)flouratheneand1,2,3-cd 
pyrene are less soluble in aqueous solution at room temperature and of higher molecular weight than the other PAHs under study. These explained reasons for the relatively delayed breakthrough time, lower breakthrough curve steepness and thus higher susceptibility to adsorption and distribution on the soil column observed during the simulation. Again, the slow movement attributed to the higher molecular PAHs could be as a result of the effects of diffusion transport mechanism dominating the migration process. The PAHs compound, particularly the higher molecular weight types, migrate slowly through large bypasses of the soil matrix by diffusion which results to delayed appearance in the effluent as portrayed by long tailings of breakthrough curves (Zhou and Wang, 2013). In relation to the present study, the structure of the PAHs could be a determinant factor for the prevailing adsorption and distribution pattern (Jacques et al., 2012). Dibenzo(a,h)anthrene,benzo(a)pyrene, Chrysene and benzo(a)anthrene are linearly fused as against the other ones which are angularly fused (Severino et al., 2010). The linearity of their structures made them soluble and less susceptible to soil column adsorption and distribution, but tend to move with the bulk flow through the soil pores (Padilla et al., 1999). Age of PAHs in the soil has been reported to affect the rapidity of desorption from the soil (ThAbdel-Moghny et al., 2012). It has been reported that Fresh Chrysene desorbes rapidly and this has been attributed to its availability in the bulk solution (Abdel-Shafy and Mansour, 2016). This attribute might explain the early breakthrough and less contact made by Chrysene. In this respect, the aforementioned PAH had little contact with soil medium, explaining the quick breakthrough time and high steep breakthrough curves observed in Fig. 2. Less biodegradable disposition, in addition to the foregoing attributes classified the aforementioned $\mathrm{PAH}$ compounds as conservatives (Eberhardt and Grathwohl, 2002; Cirpka, 2002). Migration of such compounds therefore portends great danger to integrity of groundwater quality (Olsen and Davis, 1990). These PAH compounds [Dibenzo(a,h)anthrene,benzo(a)pyrene, Chrysene and benzo(a)anthrene] are highly toxic and carcinogenic therefore raising great concern for groundwater pollution(Anderson et al., 2000).

Level of affinity of the hydrocarbons were further investigated by observing the rate of breakthrough of the compounds from the coastal sand column vis-à-vis relative concentrations and breakthrough time. Figure 3 shows varying concentration affinities of the hydrocarbons to the coastal sand. Although a considerable percentage of (Benzo(a)pyrene, Chrysene and Benzo(a)anthrene) hydrocarbons was fixed at the lower segment of the column, they were found in high concentration in the exit solution. Whereas
Benzo(b)flouranthene, benzo(K)flourathene and 1,2,3-cd pyrene were more distributed at the upper segment. This could be as a result of saturation of the soil column and apparent low affinity for Benzo(a)pyrene, Chrysene and Benzo(a)anthrene to the coastal sand, which led to quick breakthrough exhibited by the hydrocarbons. With these scenario the hydrocarbons(Benzo(a)pyrene, Chrysene andBenzo(a)anthrene) migrate fast through the soil matrix to the subsurface and perhaps compromise the groundwater quality (Johnson, 2012; Piatt et al., 1996). The other hydrocarbons, Benzo(b)flouranthene, benzo(K)flourathene and 1,2,3-cd pyrene, had delayed breakthrough and likely to remain in the soil with less migration ability.

The effects of solubility, molecular weight and structure of the compounds seemed to play out as observed previously. Benzo(b)flouranthene is Sparingly soluble in water especially at temperature within the nieghbourhood of room temperature (Hiller et al., 2008), therefore was readily available as precipitates in solution and hence sufficiently adsorbed onto the soil matrix of the upper segment $(0-4$ and $4-9 \mathrm{~cm})$ as it migrates down subsurface. The same reason could be adduced to the apparent asymmetric fixation of benzo(a)pyrene, Chrysene, 1,2,3-cd pyrene and benzo(K)flourathene on the lower segments of the column. Apart from being more soluble, the linearly fused structure of benzo(a)anthrene and Dibenzo(a,h)anthrene provided avenue for long distance transverse, thereby making substantial overall amounts remain at the lower part of the column.

Effects of molecular structure may have also contributed to the variation in amount of adsorption. The Linearly fused structure of Dibenzo(a,h)anthrene made it soluble and sparingly available in solution as precipitate for easy adsorption on the soil surface,resulting to the prevailing low adsorption. Again, 1,2,3-cd pyrene has higher molecular weight than benzo(K)flourathene yet its sorption amount was low. Here, the effects of the molecular structure of the hydrocarbons also influenced the sorption process (Lohmann, 2003). The non.-alternate arrangement of 1,2,3-cd pyrene encouraged its high solubility. This situation is predicated on the prevailing poor adsorption capacity of the hydrocarbon. The same reason also applies to values of the retardation factor, $R_{f}$, distribution coefficient, $K_{d}$ and $K_{O C}$ Distribution and affinity to organic carbons of these compounds were low due to their respective non-alternate and linearity associated with their structures. PAHs associated with low distribution coefficient and high retardation factor have the capacity to migrate with bulk flow through the pores with high possibility of increasing groundwater pollution risk. 


\section{Conclusion}

Distribution of some selected PAH compounds on a typical coastal sand deposit has been studied. Significant PAHs concentration differences were observed for different pore water volume in the process of column migration. Different PAHs sorption effects are represented on breakthrough curves. The level of PAHs sorption was measured with the relative PAHs concentrations which increased to the plateau and finally decreased as the pore water volume increased. Molecular weights, structures and solubility of the PAH compounds were key to the soil column distribution. Adsorptions and distributions of higher molecular weight, were functions of their chemical structures and solubilities. Angularly fused and less soluble PAHs adsorbed and distributed more on the soil matrix than the linearly and more soluble ones. Although a significant number of the PAH compounds was retained at the upper segment of the soil column, however, some moved with the bulk flow and were detected in the exit solution at alarming concentrations. Chances are that the ones within bulk flow have the potential to compromise the integrity of groundwater.

\section{Funding Information}

The research was self-funded.

\section{Acknowledgement}

We are grateful to the staff and management of institute of erosion, federal university of technology owerri for providing necessary assistance to analyze the soil samples. Analysis of HMW PAH compounds were carried out in the chemistry laboratory. Many thanks to all the lab technologies for their co-operations.

\section{Authors Contribution}

Uzoije Atulegwu Patrick and Ezenobi Chukwunoye C collected both the soil and water samples, preserved them for the analysis. Both also carried out the Laboratory analysis of the samples. The design and planning of the research work, reporting and typesetting were done by Atulegwu Patrick Uzoije.

\section{Ethics}

No part of this work should be reproduced without the permission of the publisher or author.

\section{References}

Abdel-Shafy, H.I. and M.S.M. Mansour, 2016. A review on polycyclic aromatic hydrocarbons: Source, environmental impact, effect on human health and remediation. Egypt. J. Petrol., 25: 107-123.

DOI: $10.1016 /$ j.ejpe.2015.03.011
Allen-King, R.M., P. Grathwohl and W.P. Ball, 2002. New modeling paradigms for the sorption of hydrophobic organic chemicals to heterogeneous carbonaceous matter in soils, sediments and rocks. Adv. Water Resources, 25: 985-1016. DOI: 10.1016/S0309-1708(02)00045-3

Anderson, P., C.M. Davison, A.L. Duncan, D. Littlejohn and A.M. Ure et al., 2000. Column leaching and sorption experiments to assess the mobility of potentially toxic elements in industrially contaminated land. J. Environ. Monitor., 23: 234239. DOI: $10.1039 / \mathrm{a} 909419 \mathrm{i}$

Benekos, I.D., O.A. Cirpka and P.K. Kitanidis, 2006. Experimental determination of transverse dispersivity in a helix and a cochlea. Water Resour. Res. DOI: 10.1029/2005WR004712

Chen, J., X.J. Wang, J.D. Hu and S.O. Tao, 2006. Modeling surfactant LAS influenced PAHs migration in soil column. Air Water Soil Pollut., 176: 217-232. DOI: 10.1007/s11270-006-9164-4

Chiou, C.T., S.E. McGroddy and D.E. Kile, 1998. Partition characteristics of polycyclic aromatic hydrocarbons on soils and sediments. Environ. Sci. Technol., 32: 264-269. DOI: 10.1021/es970614c

Christensen, E.R. and S. Arora, 2006. Source apportionment of PAHs in sediments using factor analysis by time records: Application to lake Michigan, USA. Water Res., 23: 1-8. DOI: 10.1016/j.watres.2006.09.009

Cirpka, O.A., 2002. Choice of dispersion coefficients in reactive transport calculations on smoothed fields. J. Contam. Hydrol., 58: 261-282. DOI: $10.1016 / \mathrm{S} 0169-7722(02) 00039-6$

Eberhardt, C. and P. Grathwohl, 2002. Time scales of organic contaminant dissolution from complex source zones: coal tar pools Vs. blobs. J. Contam. Hydrol., 59: 45-66.

DOI: 10.1016/S0169-7722(02)00075-X

Edwards, N.T., 1983. Polycyclic Aromatic Hydrocarbons (PAH's) in the terrestrial environment-A review. J. Environ. Quality, 12: 427-441. DOI: $10.2134 /$ jeq1983.00472425001200040001x

Haws, N., B.S. Das and P.S.C. Rao, 2004. Dual-domain solute transfer and transport processes: Evaluation in batch and transport experiments. J. Contam. Hydrol., 75: 24-32.

DOI: 10.1016/j.jconhyd.2004.07.001

He, Y., J. Xu, H. Wang, Z. Ma and J. Chen, 2006. Detailed sorption isotherms of pentachlorophenol on soils and its correlation with soil properties. Environ. Res., 101: 362-372. DOI: $10.1016 /$ j.envres.2006.01.002

Hiller, E., L. Jurkovič and M. Bartal, 2008. Effect of temperature on the distribution of polycyclic aromatic hydrocarbons in soil and sediment. Soil Water Res., 3: 231-240. 
Hwang, S. and T.J. Cutright, 2002. The impact of contact time on pyrene sorptive behavior by a sandy-loam soil. Environ. Poll., 117: 371-378. DOI: $10.1016 / \mathrm{S} 0269-7491(01) 00202-0$

Hwang, S., N. Ramirez, T.J. Cutright and L.K. Ju, 2003. The role of soil properties in pyrene sorption and desorption. Water Air Soil Pollut., 143: $65-80$.

DOI: $10.1023 / \mathrm{A}: 1022863015709$

Jacques, D., C. Smith, J. Šimůnek and D. Smiles, 2012. Inverse optimization of hydraulic, solute transport and cation exchange parameters using HP1 and UCODE to simulate cation exchange. J. Contaminant Hydrol., 142-143: 109-125. DOI: $10.1016 /$ j.jconhyd.2012.03.008

Johnson, R.O., 2012. Analytical modeling of linear migration. Groundwater Int. Scholarly Res. Netw. Chem. Eng., 2012: 32-32

Joo, J.C., C.D. Shackelford and K.F. Reardon, 2008. Sorption of nonpolar neutral organic compounds to humic acid-coated sands: Contributions of organic and mineral components. Chemosphere, 70: $1290-1297$.

DOI: 10.1016/j.chemosphere.2007.07.052

Kim, J.Y. and M. Osako, 2003. Leaching characteristics of Polycyclic Aromatic Hydrocarbons (PAHs) from spiked sandy soil. Chemosphere, 51: 387-395. DOI: $10.1016 / \mathrm{S} 0045-6535(02) 00866-4$

Lee, L.S., P.S. Rao and I. Okuda, 1992. Equilibrium partitioning of polycyclic aromatic hydrocarbons from coal tar into water. Environ. Sci. Technol., 26: 2110-2115. DOI: $10.1021 / \mathrm{es} 00035 \mathrm{a} 006$

Lohmann, R., 2003. The emergence of black carbon as a super-sorbent in environmental chemistry: The end of Octanol: Environ. Forens., 4: 161-165. DOI: 10.1080/713848510

Ma, C., Y. Wu, C. Sun and L. Lee, 2007. Adsorption characteristics of perchloroethylene in natural sandy materials with low organic carbon content. Environ. Geol., 52: 1511-1519.

DOI: $10.1007 / \mathrm{s} 00254-006-0595-4$

Nguyen, T.H. and W.P. Ball, 2006. Absorption and adsorption of hydrophobic organic contaminants to diesel and hexane soot. Environ. Sci. Technol., 40: 2958-2964. DOI: 10.1021/es052121a

O'Reilly, K.T., R.I. Magaw and W.G. Rixey, 2001. Predicting the effects of hydrocarbon and hydrocarbon impacted soil on groundwater. Am. Petrol. Inst., 14: 23-31.

Olsen, R.L. and A. Davis, 1990. Predicting the fate and transport of organic compounds in groundwater Part 1. Hazardous Mater. Control, 3: 38-64.
Ortega-Calvo, J.J., M. Lahlou and C. Saiz-Jimenez, 1997. Effect of organic matter and clays on the biodegradation of phenanthrene in soils. Int. Biodet. Biodeg., 40: 101-106. DOI: $10.1016 / \mathrm{S} 0964-8305(97) 00045-0$

Padilla, I.Y., T.C.J. Yeh and M.H. Conklin, 1999. The effect of water content on solute transport in unsaturated porous media. Water Resources Res., 35: 3303-3313. DOI: 10.1029/1999WR900171

Park, H.S. and C.S. Juan, 2000. A method for assessing leaching potential for petroleum hydrocarbons release sites: Multiphase and multisubstance equilibrium partitioning. J. Soil Contaminat., 9: 611-632. DOI: 10.1080/10588330091134437

Patrick, U.A. and U. Cosmas, 2016. Studying and modeling dynamic adsorption of lead (II) ion onto fixed bed column of activated carbon of plantain peels and bamboo. Sci. J. Environ. Eng. Res., 2016: 115-115. DOI: $10.7237 /$ sjeer/115

Piatt, J.J., D.A. Backhus, P.D. Capel and S.J. Eisenreich, 1996. Temperature-dependent sorption of naphthalene, phenanthrene and pyrene to low organic carbon aquifer sediments. Environ. Sci. Technol., 30: 751-760. DOI: 10.1021/es9406288

Severino, G., A. Comegna, A. Coppola, A. Sommella and A. Santini, 2010. Stochastic analysis of a fieldscale unsaturated transport experiment. Adv. Water Resources, 33: 1188-1198.

DOI: $10.1016 /$ j.advwatres.2010.09.004

ThAbdel-Moghny, T., R.S.A. Mohamed, E. El-Sayed, S.M. Aly and M.G. Snousy, 2012. Effect of soil texture on remediation of hydrocarbonscontaminated soil at el-Minia district, Upper Egypt. ISRN Chem. Eng., 2012: 406598-406598. DOI: $10.5402 / 2012 / 406598$

Toul, J., J. Bezděk, M. Kovářová, Z. Boháček and J.Hanák et al., 2003. Sorption of hydrophobic organic pollutants on soils and sediments. Bull. Geosci., 78: 205-223.

USEPA, 1990. Chemical fate rate constantsfor SARA section 113 chemicals and superfund health evaluation Manual Chemicals. United States Environmental Protection Agency, Office of Toxic Substances, Washington DC.

Uzoije, A.P., C.O. Kamalu and U. Basil, 2015. Adsorption kinetics and mechanisms of cypermethrin and dichlorovos on heterogeneous activated carbon porous media. Open Access Library J., 2: e1988-e1988.

DOI: $10.4236 /$ oalib. 1101988

van Genuchten, M.T. and R.J. Wagenet, 1989. Twosite/two-region models for pesticide transport and degradation: Theoretical development and analytical solutions. Soil Sci. Soc. Am. J., 53: 1303-1310. DOI: $10.2136 /$ sssaj $1989.03615995005300050001 \mathrm{x}$ 
Weber, W.J., P.M. McGinley and L.E. Katz, 1992. Sorption phenomena in subsurface systems: Concepts, models and effects on contaminant fate and transport. Environ. Sci. Technol., 26: 1955-1962.

Yaron, B., R. Calvet and R. Prost, 1996. Soil Pollution: Processes and Dynamics. 1st Edn., Springer Science and Business Media, Berlin,

ISBN-10: 354060927X, pp: 313.
Zhou, B.B. and Q.J. Wang, 2013. Effect of pore water velocities and solute input methods on chloride transport in the undisturbed soil columns of Loess Plateau. Applied Water Sci., 7: 2321-2328.

DOI: $10.1007 / \mathrm{s} 13201-016-0408-1$ 